\title{
Effects of cosmic-string framework on the thermodynamical properties of anharmonic oscillator using the ordinary statistics and the $q$-deformed superstatistics approaches
}

\author{
Hadi Sobhani $^{1, a}$, Hassan Hassanabadi ${ }^{1}$, Won Sang Chung ${ }^{2}$ \\ ${ }^{1}$ Faculty of Physics, Shahrood University of Technology, P. O. Box 3619995161-316, Shahrood, Iran \\ ${ }^{2}$ Department of Physics and Research Institute of Natural Science, College of Natural Science, Gyeongsang National University, Jinju 52828, \\ Korea
}

Received: 21 July 2017 / Accepted: 23 January 2018 / Published online: 6 February 2018

(C) The Author(s) 2018. This article is an open access publication

\begin{abstract}
In this article, we determine the thermodynamical properties of the anharmonic canonical ensemble within the cosmic-string framework. We use the ordinary statistics and the $q$-deformed superstatistics for this study. The $q$-deformed superstatistics is derived by modifying the probability density in the original superstatistics. The Schrödinger equation is rewritten in the cosmic-string framework. Next, the anharmonic oscillator is investigated in detail. The wave function and the energy spectrum of the considered system are derived using the bi-confluent Heun functions. In the next step, we first determine the thermodynamical properties for the canonical ensemble of the anharmonic oscillator in the cosmic-string framework using the ordinary statistics approach. Also, these quantities have been obtained in the $q$ deformed superstatistics. For vanishing deformation parameter, the ordinary results are obtained.
\end{abstract}

\section{Introduction}

Topological defects were first theorized by Kibble [1] These defects are physical structures produced in symmetrybreaking phase transitions in the early universe. Among all the possible types of defects, the one-dimensional cosmic strings are the focus of most of the studies in this area [2]. This is because of the compatibility with the current cosmological models and their association with several brane inflation scenarios [3,4] and super-symmetric grand unified theories [5]. These are constrained by the cosmic microwave background [6-10] and gravitational wave facilities [11-15]. In recent developments, cosmic strings have been considered to study solutions of black holes [16], to investigate the average rate of change of energy for a static atom immersed in a thermal bath of electromagnetic radiation [17], to study Hawking radiation of massless and massive charged particles [18], to study the non-Abelian Higgs model coupled with gravity [19], in the quantum dynamics of scalar bosons [20], in hydrodynamics [21], to study the non-relativistic motion of a quantum particle subjected to magnetic field [22], to investigate dynamical solutions in the context of super-critical tensions [23], describing scattering states of the Dirac equation in the presence of cosmic string for Coulomb interaction [24] and to study the spin-zero Duffin-Kemmer-Petiau equation in a cosmic-string space-time with the Cornell interaction [25].

The concept of superstatistics was initiated by Beck and Cohen [26]. They considered non-equilibrium systems with complex dynamics in stationary states with large fluctuations of intensive quantities on large time scales. Depending on the statistical properties of the fluctuations, they obtain different effective statistical mechanical descriptions. To derive and compute macroscopic quantities such as the Helmholtz free energy, the entropy, and the mean energy, the Boltzmann factor $e^{\beta E}$ is the essential tool; here $\beta=\frac{1}{k_{B} T}$ with $k_{B}$ being the Boltzmann constant and $T$ denoting the temperature. But this condition no longer will exist when the equilibrium conditions are lost. As a result, therefore, we need to define an effective Boltzmann factor $B(E)$ as

$B(E)=\int_{0}^{\infty} e^{\beta^{\prime} E} f\left(\beta_{0}^{\prime}, \beta\right) \mathrm{d} \beta^{\prime}$

where $f\left(\beta^{\prime}, \beta\right)$ is a probability density and, according to the definition (1.1), this is an average of the ordinary Boltzmann factor. Referring to Ref. [26], the probability density must obey the conditions:

\footnotetext{
a e-mail: hadisobhani8637@gmail.com
} 
- The probability density should be non-negative and

$$
\int_{0}^{\infty} f\left(\beta_{0}^{\prime}, \beta\right) \mathrm{d} \beta^{\prime}=1
$$

- The average of $\beta_{0}^{\prime}$ should be $\beta=\frac{1}{k_{B} T}$

$$
\left\langle\beta_{0}^{\prime}\right\rangle=\int_{0}^{\infty} \beta_{0}^{\prime} f\left(\beta_{0}^{\prime}, \beta\right) \mathrm{d} \beta_{0}^{\prime}=\beta
$$

- The superstatistics must be normalizable. For instance the integral $\int_{0}^{\infty} B(E) \mathrm{d} E$ should exist and, in the general case, the integral $\int_{0}^{\infty} \rho_{d}(E) B(E) \mathrm{d} E$ has to exist in which $\rho_{d}(E)$ is the density of states.

- The superstatistics, if there are no fluctuations of intensive quantities, should reduce to the Boltzmann-Gibbs statistic.

If the probability density function has been considered as

$f\left(\beta_{0}^{\prime}, \beta\right)=\delta\left(\beta_{0}^{\prime}-\beta\right)$

causes to have the following effective Boltzmann factor:

$B(E)=e^{-\beta E}$

In this paper, we first intend to modify the probability density and discuss the thermodynamical proprieties of the anharmonic oscillator ensemble within the cosmic-string framework. Then, in Sect. 2, the probability density is modified and the new effective Boltzmann factor is derived. In Sect. 3, the Schrödinger equation within the cosmic string is derived. The anharmonic oscillator has been studied in detail by deriving the wave function and its energy spectrum in Sect. 4. After derivation of the wave function and the energy spectrum, the thermodynamical properties of the anharmonic oscillator ensemble are derived in two subsections. In the first subsection of Sect. 5, considering the ordinary probability density for $f\left(\beta_{0}^{\prime}, \beta\right)$, thermodynamical properties of the ensemble considered have been obtained. In the other subsection, considering the modified form of the probability density we will obtain thermodynamical properties of the system. Since we have performed a mathematical physics development of the topic, we have obtained the general results such that in all cases, when the modification parameter is removed, ordinary results will be derived.

\section{Modified of probability density}

As regards superstatistics, the standard Boltzmann statistics is related to the probability density (1.4), which results in the Boltzmann factor (1.5). There are four conditions for the effective Boltzmann factor. The average of $\beta^{\prime}$ is $\beta$ with zero variance indicating this point that there is no fluctuation for $\beta^{\prime}$ due to the zero variance. Now, we want to consider a general case in which the fluctuations can be explained by the nonvanishing variance. Let us consider the modified form of the probability density [27],

$$
\begin{aligned}
f\left(\beta_{0}^{\prime}, \beta\right)= & a_{1} \delta\left(\beta_{0}^{\prime}-\beta\right)+b_{1} \beta_{0}^{\prime} \frac{\partial}{\partial \beta_{0}^{\prime}} \delta\left(\beta_{0}^{\prime}-\beta\right) \\
& +c_{1} \beta_{0}^{\prime 2} \frac{\partial}{\partial \beta_{0}^{\prime 2}} \delta\left(\beta_{0}^{\prime}-\beta\right),
\end{aligned}
$$

where $a_{1}, b_{1}, c_{1}$ are real constants that shall be determined. It is well known that the Dirac delta function obeys the following relations:

$$
\int_{0}^{\infty} \delta\left(\beta_{0}^{\prime}-\beta\right) \mathrm{d} \beta_{0}^{\prime}=1
$$

$\int_{0}^{\infty} \delta\left(\beta_{0}^{\prime}-\beta\right) F\left(\beta_{0}^{\prime}\right) \mathrm{d} \beta_{0}^{\prime}=F(\beta)$,

$\int_{0}^{\infty}\left(\frac{\partial}{\partial \beta_{0}^{\prime}} \delta\left(\beta_{0}^{\prime}-\beta\right)\right) F\left(\beta_{0}^{\prime}\right) \mathrm{d} \beta_{0}^{\prime}=-\frac{\partial}{\partial \beta} F(\beta)$,

$\int_{0}^{\infty}\left(\frac{\partial^{2}}{\partial \beta_{0}^{\prime 2}} \delta\left(\beta_{0}^{\prime}-\beta\right)\right) F\left(\beta_{0}^{\prime}\right) \mathrm{d} \beta_{0}^{\prime}=\frac{\partial^{2}}{\partial^{2} \beta} F(\beta)$.

Using these properties, Eqs. (1.2), (1.3) and considering the variance of such a distribution to be $q \beta^{2}$ with $q>0$ we have

$a_{1}-b_{1}+2 c_{1}=1$,

$a_{1}-2 b_{1}+6 c_{1}=1$,

$a_{1}-3 b_{1}+12 c_{1}=q+1$.

By solving the above equations, we have the explicit form of the modified probability density as

$$
\begin{aligned}
f\left(\beta_{0}^{\prime}, \beta\right)= & (1+q) \delta\left(\beta_{0}^{\prime}-\beta\right)+2 q \beta_{0}^{\prime} \frac{\partial}{\partial \beta_{0}^{\prime}} \delta\left(\beta_{0}^{\prime}-\beta\right) \\
& +\frac{q}{2} \beta_{0}^{\prime 2} \frac{\partial}{\partial \beta_{0}^{\prime 2}} \delta\left(\beta_{0}^{\prime}-\beta\right)
\end{aligned}
$$

Consequently, this distribution has $\beta$ and $q \beta^{2}$ as mean and variance, respectively. According to the definition of (1.1), we have the effective Boltzmann factor as

$B(E)=\int_{0}^{\infty} e^{-\beta_{0}^{\prime} E} f\left(\beta_{0}^{\prime}, \beta \mathrm{d} \beta_{0}^{\prime}\right)=e^{\beta E}\left(1+\frac{q}{2} \beta^{2} E^{2}\right)$.

It is seen that by removing the modification parameter, $q \rightarrow 0$, ordinary results will be recovered. Now, we can discuss the Schrödinger equation within cosmic-string frame- 
work considering the anharmonic oscillator to obtain thermodynamical properties.

\section{Schrödinger equation within cosmic-string framework}

In the cosmic-string framework without internal structure, the metric is [28]

$$
\mathrm{d} s^{2}=-(c \mathrm{~d} t+a \mathrm{~d} \phi)^{2}+\mathrm{d} \rho^{2}+\alpha^{2} \rho^{2} \mathrm{~d} \phi^{2}+\mathrm{d} z^{2}
$$

in which the angular parameter is associated to cosmic string space-time represented by $\alpha$, that is, $\alpha \leq 1, a=\frac{4 G J}{c^{3}}$ indicates the spinning parameter of the cosmic string and $J$ denotes the linear mass density of the cosmic string. The time-dependent component of the metric can be neglected if the coupling between angular momentum of the particle and the angular momentum of the cosmic string is supposed to be very weak [28]. The metric tensor of the considered curvilinear frame can be determined as

$g_{i j}=\left(\begin{array}{ccc}1 & 0 & 0 \\ 0 & \alpha^{2} \rho^{2}-a^{2} & 0 \\ 0 & 0 & 1\end{array}\right)$.

We use the general definition of the Laplacian,

$\nabla^{2}=\frac{1}{\sqrt{g}} \partial_{i}\left(\sqrt{g} g^{i j} \partial_{j}\right)$,

where $g=\operatorname{det}\left(g^{i j}\right)=\alpha^{2} \rho^{2}-a^{2}$. According to the definition of Laplacian 2.3 and the tensor metric, we have

$\nabla^{2}=\frac{\partial^{2}}{\partial \rho^{2}}+\frac{\alpha^{2} \rho}{\alpha^{2} \rho^{2}-a^{2}} \frac{\partial}{\partial \rho}+\left(\alpha^{2} \rho^{2}-a^{2}\right) \frac{\partial^{2}}{\partial \phi^{2}}+\frac{\partial^{2}}{\partial z^{2}}$.

Thus, the Hamiltonian can be written as $(\hbar=c=1)$

$$
\begin{aligned}
H= & -\left[\frac{\partial^{2}}{\partial \rho^{2}}+\frac{\alpha^{2} \rho}{\alpha^{2} \rho^{2}-a^{2}} \frac{\partial}{\partial \rho}\right. \\
& \left.+\left(\alpha^{2} \rho^{2}-a^{2}\right) \frac{\partial^{2}}{\partial \phi^{2}}+\frac{\partial^{2}}{\partial z^{2}}\right]+V(\rho, \phi, z) .
\end{aligned}
$$

where we have supposed $m=0.5$. Now we can consider a two-dimensional interaction term.

\section{The anharmonic oscillator}

The anharmonic oscillator is written as [29-31]

$$
v(\rho)=a_{v} \rho^{2}+b_{v} \rho^{4}+c_{v} \rho^{6},
$$

where the coefficients are real constants. Such a potential can be used in nuclear physics and the expressions of the energy rates and quadrapole transitions. In Ref. [29], the authors used the series expansion and tried to derive recurrence relation between the coefficients of the series or the Gaussian approximation to obtain the result in Ref. [30]; the authors used asymptotic iteration method to obtain the solutions. However, we used some changes of variables to derive the bi-confluent Huen differential equations [31]. Substituting (4.1) into (3.5) for $\rho \geq \frac{a}{\alpha}$ and considering the wave function $\Psi(t, \mathrm{r})=\exp [i(-\varepsilon t+l \phi+k z)] \Phi(\rho)$ we obtain

$$
\begin{aligned}
& \frac{\mathrm{d}^{2} \Phi(\rho)}{\mathrm{d} \rho^{2}}+\frac{1}{\rho} \frac{\mathrm{d} \Phi(\rho)}{\mathrm{d} \rho}+\left(\varepsilon-k^{2}-\left(a_{v}+l^{2} \alpha^{2}\right) \rho^{2}\right. \\
& \left.-b_{v} \rho^{4}-c_{v} \rho^{6}\right) \Phi(\rho)=0 .
\end{aligned}
$$

To solve Eq. (4.2), first derivative terms should be removed by considering $\Phi(\rho)=\frac{R(\rho)}{\sqrt{\rho}}$. Then we obtain

$$
\begin{aligned}
& \frac{\mathrm{d}^{2} R(\rho)}{\mathrm{d} \rho^{2}}+\left(\varepsilon+\frac{1}{4 \rho^{2}}-k^{2}-\left(a_{v}+l^{2} \alpha^{2}\right) \rho^{2}\right. \\
& \left.-b_{v} \rho^{4}-c_{v} \rho^{6}\right) R(\rho)=0 .
\end{aligned}
$$

The next step is using the new variable $y=\rho^{2}$. Rewriting Eq. (4.3) in terms of the new variable, results in

$$
\begin{aligned}
& \frac{\mathrm{d}^{2} R(y)}{\mathrm{d} y^{2}}+\frac{1}{2 y} \frac{\mathrm{d} R(y)}{\mathrm{d} y}+\left(\frac{1}{16 y}+\frac{\frac{1}{4}\left(\varepsilon-k^{2}\right)}{y}-\frac{1}{4}\left(a_{v}+l^{2} \alpha^{2}\right)\right. \\
& \left.-\frac{b_{v}}{4} y-\frac{c_{v}}{4} y^{2}\right) R(y)=0 .
\end{aligned}
$$

Once again, we need to remove the first derivative term in Eq. (4.4) to continue to get to the solutions. This time we consider $R(y)=\frac{f(y)}{\sqrt[4]{y}}$ and obtain

$$
\begin{aligned}
& \frac{\mathrm{d}^{2} f(y)}{\mathrm{d} y^{2}}+\left(\frac{1}{4 y}+\frac{\frac{1}{4}\left(\varepsilon-k^{2}\right)}{y}-\frac{1}{4}\left(a_{v}+l^{2} \alpha^{2}\right)\right. \\
& \left.-\frac{b_{v}}{4} y-\frac{c_{v}}{4} y^{2}\right) f(y)=0 .
\end{aligned}
$$

Considering the solution in the form of

$$
f(y)=y_{1}^{A} \exp \left[y\left(B_{1}+D_{1} y\right)\right] F(y),
$$

where

$$
A_{1}=\frac{1}{2},
$$


$B_{1}=-\frac{b_{v}}{8}$,

$D_{1}=-\frac{\sqrt{c_{v}}}{2}$

we have

$$
\begin{aligned}
& \frac{\mathrm{d}^{2} F(y)}{\mathrm{d} y^{2}}+\left(\frac{1}{y}-\frac{b_{v}}{4}-2 y\right) \frac{\mathrm{d} F(y)}{\mathrm{d} y} \\
& \quad+\left(\frac{b_{v}^{2}}{64}-\frac{1}{4}\left(a_{v}^{2}+l^{2} \alpha^{2}+4+\frac{-\frac{b_{v}}{8}+\frac{1}{4}\left(\varepsilon-k^{2}\right)}{y}\right)\right) \\
& F(y)=0,
\end{aligned}
$$

in which we were forced to set $c_{v}=4$ (see the appendix for more details). Equation (4.10) is the bi-confluent Heun differential equation. Consequently, we have

$$
\begin{aligned}
& F(y)=H_{b}\left(0, \beta_{b}^{\prime}, \gamma_{b}^{\prime}, \delta_{b}^{\prime} ; y\right), \\
& \beta_{b}^{\prime}=\frac{b_{v}}{4}, \\
& \gamma_{b}^{\prime}=\frac{b_{v}^{2}}{4}-\frac{1}{4}\left(a_{v}^{2}+l^{2} \alpha^{2}-4\right), \\
& \delta_{b}^{\prime}=\frac{1}{2}\left(k^{2}-\varepsilon\right) .
\end{aligned}
$$

To obtain the energy spectrum of the considered system, we should use expansion of bi-confluent Heum functions. Thus, considering $F(y)=\sum_{n=0}^{\infty} s_{n} y^{n}$, we obtain the constraints

$-\beta_{b}^{\prime}(n+2)=\frac{\delta_{b}^{\prime}+\beta_{b}^{\prime}}{2}$,

$4+2 n=\gamma_{b}^{\prime}$.

Using these constraints and Eqs. (4.11), (4.12) and (4.13) we have

$\varepsilon=\frac{b_{v}}{2}(2 n+5)+k^{2}$,

$b_{v}= \pm \sqrt{12+8 n+a_{v}^{2}+l^{2} \alpha^{2}}$.

Therefore, the final form of radial wave function can be written as

$\phi(\rho)=N_{c} \exp \left(-\rho^{2}\left(\frac{b_{v}}{8}+\frac{\rho^{2}}{2}\right)\right) H_{b}\left(0, \beta_{b}^{\prime}, \gamma_{b}^{\prime}, \delta_{b}^{\prime} ; \rho^{2}\right)$,

where $N_{c}$ is the normalization constant. As was proved, we have only one free parameter. Figure 1 depicts the behavior of the potential. Figure 2 illustrates the square of the wave function norm vs. various alpha values.
Now the wave function and energy spectrum relation are determined. Thus we are ready to investigate some thermodynamical properties from a statistical mechanics point of view.

\section{Thermodynamical properties within cosmic-string framework}

In this section, the thermodynamical properties of the considered system are investigated in two manners. In the first subsection of this section, we want to study thermodynamical properties such as the entropy, the Helmholtz free energy, the mean energy and the entropy. In the other subsection, these properties are investigated in the $q$-deformed superstatistics manner.

\subsection{Thermodynamical properties; ordinary statistics approach}

To derive thermodynamical quantities from a statistical mechanical point of view, we should construct the partition function. To construct the partition function we have $(\hbar=1, m=0.5)$

$$
\begin{aligned}
Z\left(\varepsilon_{n}\right) & =\sum_{n=0}^{\infty} e^{-\beta \varepsilon_{n}} \\
& =\sum_{n=0}^{\infty} e^{-\beta\left(\left(b_{v} n+\frac{5 b_{v}}{2}\right)+k^{2}\right)} \\
& =e^{-\beta\left(\frac{5 b_{v}}{2}+k^{2}\right)} \sum_{n=0}^{\infty} e^{-\beta b_{v} n} \\
Z\left(\varepsilon_{n}\right) & =\frac{e^{-\beta\left(\frac{5 b_{v}}{2}+k^{2}\right)}}{1-e^{-\beta b_{v}}},
\end{aligned}
$$

where $\beta=\frac{1}{k_{B} T}, k_{B}$ is the Boltzmann constant and the temperature is denoted $T$. In Fig. 3, we have plotted the partition function in terms of $\alpha$. It is seen that by increasing the value of $\alpha$, the partition function decreases.

As the first quantity, the Helmholtz free energy can be obtained using Eq. (5.1) as

$A=\frac{-1}{\beta} \ln Z\left(\varepsilon_{n}\right)$,

$A=\frac{-1}{\beta} \ln \left(\frac{e^{-\beta \frac{3 b_{v}+2 k^{2}}{2}}}{e^{\beta b_{v}}-1}\right)$.

In Fig. 4, we have plotted Helmholtz free energy as a function of $\alpha$. Effect of the $\alpha$ parameter can be seen that by increasing in the $\alpha$ parameter, the Helmholtz free energy increases too. 

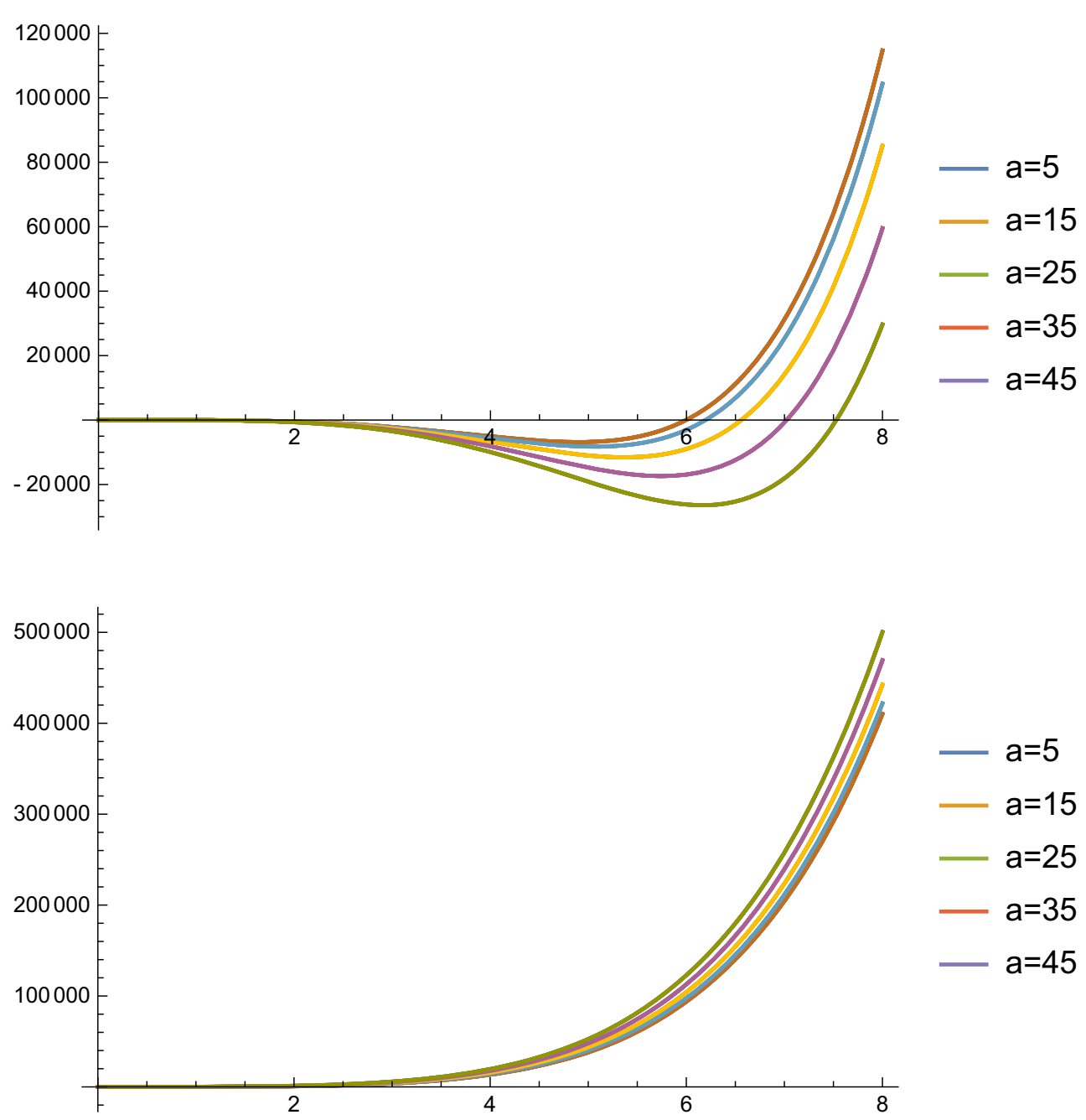

Fig. 1 Treatments of the anharmonic potential. The first plot is for the minus sign of $b$ and the second plots is for the positive sign of $b$

We can obtain from the Helmholtz free energy the entropy. According to its definition, we have

$S=-\frac{\partial A}{\partial T}$,

$S=\frac{1}{2 T}\left(2 k^{2}+b\left(5+\frac{2}{e^{\beta b_{v}}-1}\right)\right)+k_{B} \ln \left(\frac{e^{-\beta \frac{3 b_{v}+2 k^{2}}{2}}}{e^{\beta b_{v}}-1}\right)$.

It is instructive to investigate the entropy behavior as a function of $\alpha$. In Fig. 2, we showed that by increasing $\alpha$, the density probability of the wave function will be concentrated. Thus we expect such a treatment for the entropy. As is shown in Fig. 5, by increasing $\alpha$, the entropy will decrease. It means that we are facing with a system that is becoming more ordered than before by increasing $\alpha$.

The mean energy is the next quantity that we want to derive. Using the connection between the mean energy and the partition function we can write
$U=A-T\left(\frac{\partial A}{\partial T}\right)$

$U=k^{2}+b\left(\frac{5}{2}+\frac{1}{e^{\beta b_{v}}-1}\right)$.

Unlike the entropy, the mean energy increases with increasing $\alpha$. Figure 6, shows such a treatments.

As the last quantity which we want to know as regards treatment in the cosmic-string framework, we have the specific heat capacity at constant volume. This quantity can be connected to the mean energy by

$$
\begin{aligned}
C_{V} & =\left(\frac{\partial U}{\partial T}\right), \\
C_{V} & =\frac{b^{2}}{4 k_{B} T^{2}} \operatorname{Csch}\left(2 \beta b_{v}\right) .
\end{aligned}
$$

As expected from the form of the entropy, we have an ensemble with low entropy, consequently the specific heat capacity 


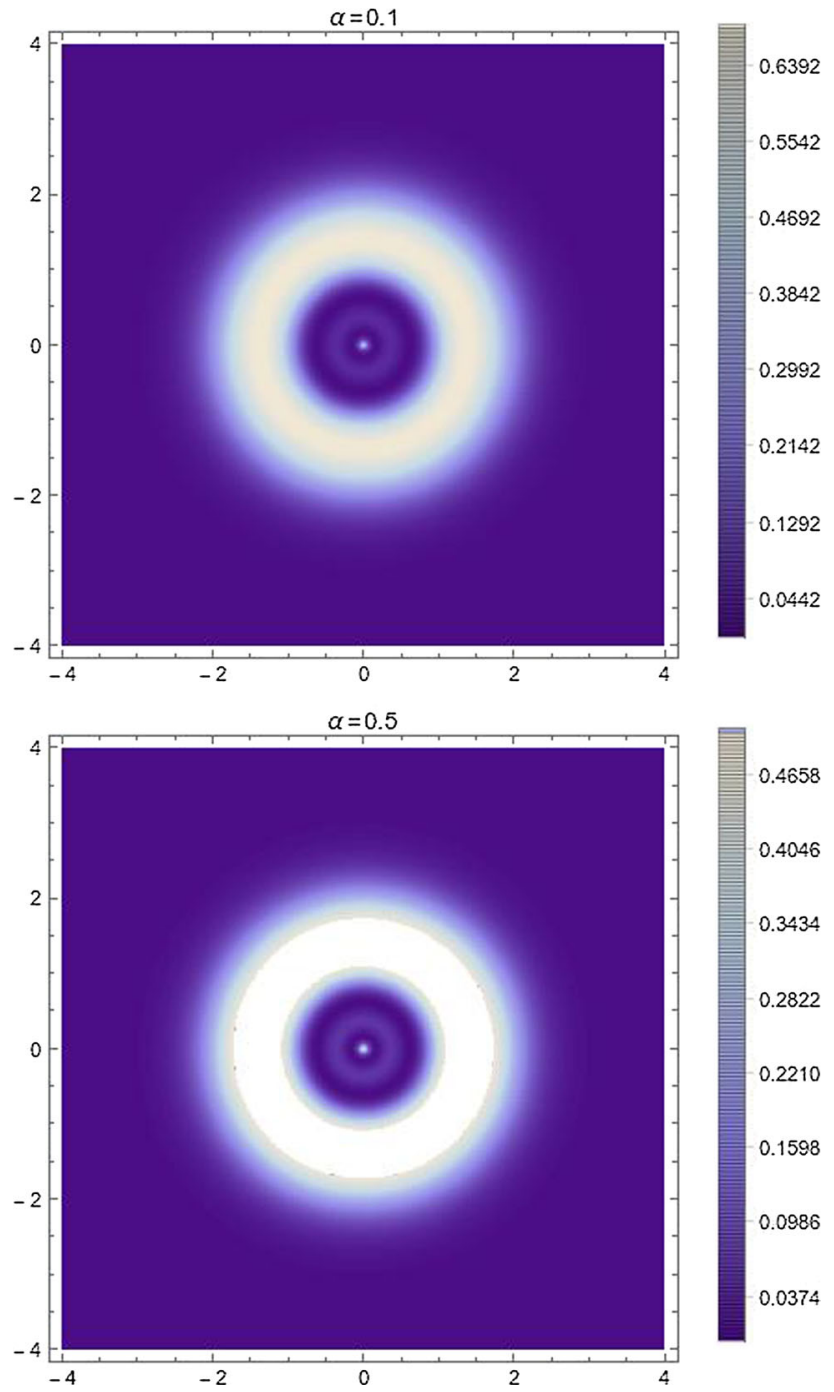

Fig. 2 Effects of $\alpha$ on $|\Psi(\rho)|^{2}$ considering $n=3, l=1, \alpha=0.2, a=$ $1 ;$ and $k=1$

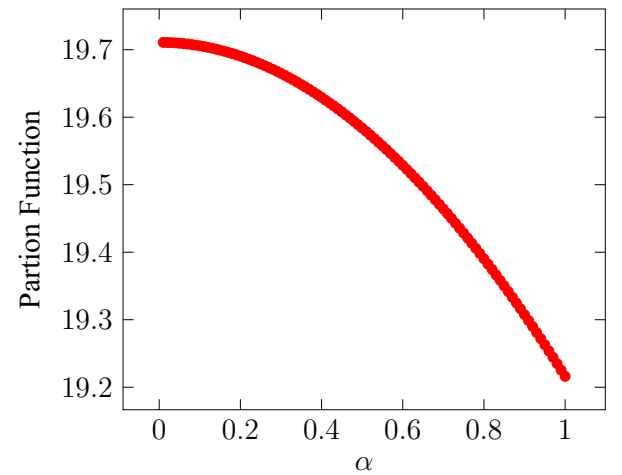

Fig. 3 Partition function vs. $\alpha$ considering $n=1, l=1, k=1, k_{B}=$ $1, a=1$ and $T=100$

at constant volume must have a lower value than an ensemble which has higher entropy. Figure 7 shows that, by increasing $\alpha$, the specific heat capacity at constant volume decreases.

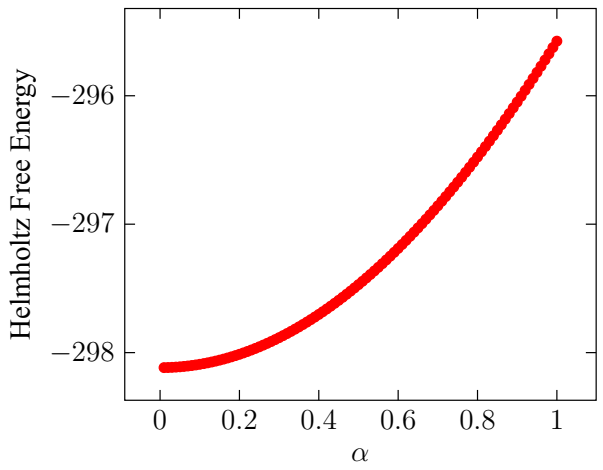

Fig. 4 Helmholtz free energy vs. $\alpha$ considering $n=1, l=1, k=$ $1, k_{B}=1, a=1$ and $T=100$

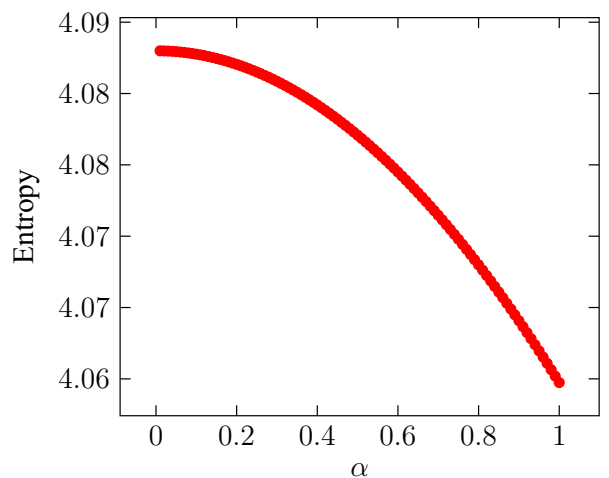

Fig. 5 Entropy vs. $\alpha$ considering $n=1, l=1, k=1, k_{B}=1, a=1$ and $T=100$

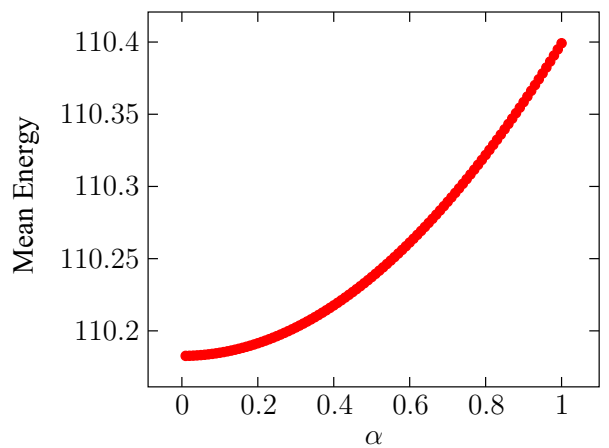

Fig. 6 Mean energy vs. $\alpha$ considering $n=3, l=1, k=1, k_{B}=$ $1, a=1$ and $T=100$

\subsection{Thermodynamical properties; $q$-deformed superstatistics approach}

The first step is the derivation of the partition function, which takes the form 


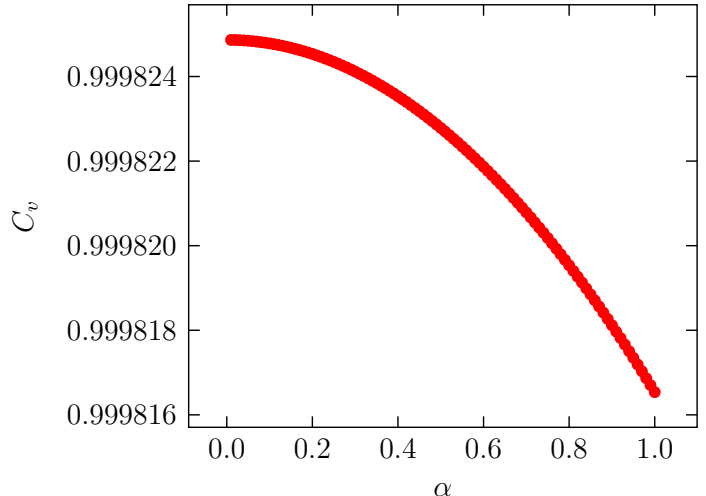

Fig. 7 Specific heat energy at constant volume vs. $\alpha$ considering $n=$ $1, l=1, k=1, k_{B}=1, a=1$ and $T=100$

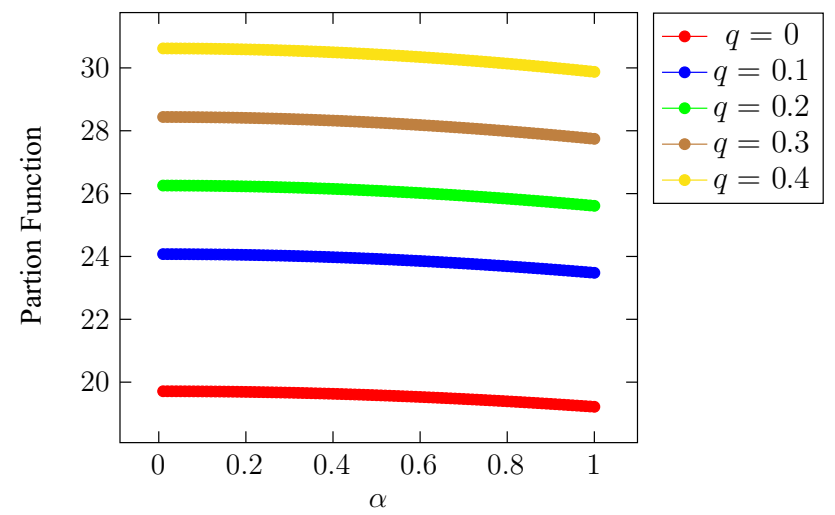

Fig. 8 Partition functions vs. $\alpha$ in the $q$-deformed superstatistics case considering $n=1, l=1, k=1, k_{B}=1, a=1$ and $T=100$ for different values of the deformation parameter

$Z(\varepsilon)=\frac{e^{-\frac{3 b_{v}+2 k^{2}}{2 T k_{B}}}\left(q \Theta(T)+8 T^{2} k_{B}^{2}\left(e^{\frac{b_{v}}{T k_{B}}}-1\right)^{2}\right)}{8 T^{2} k_{B}^{2}\left(e^{\frac{b_{v}}{T k_{B}}}-1\right)^{3}}$

where

$$
\begin{aligned}
\Theta(T)= & -2\left(13 b_{v}^{2}+16 b_{v} k^{2}+4 k^{4}\right) e^{\frac{b_{v}}{T k_{B}}} \\
& +\left(5 b_{v}+2 k^{2}\right)^{2} e^{\frac{2 b_{v}}{T k_{B}}}+\left(3 b_{v}+2 k^{2}\right)^{2} .
\end{aligned}
$$

After determination of the partition function, we can obtain the Helmholtz free energy, entropy, mean energy, and specific heat at constant volume. The behaviors of these quantities are depicted in Figs. 8, 9,10, 11 and 12. We studied the presence $(q \neq 0)$ and absence $(q=0)$ of the modification. As we stated in Sect. 2, $q$ is a positive constant. Consequently, we can see that effects of the deformation parameter on the quantities is increasing in their values. The important effect of the deformation parameter is that in the absence of the

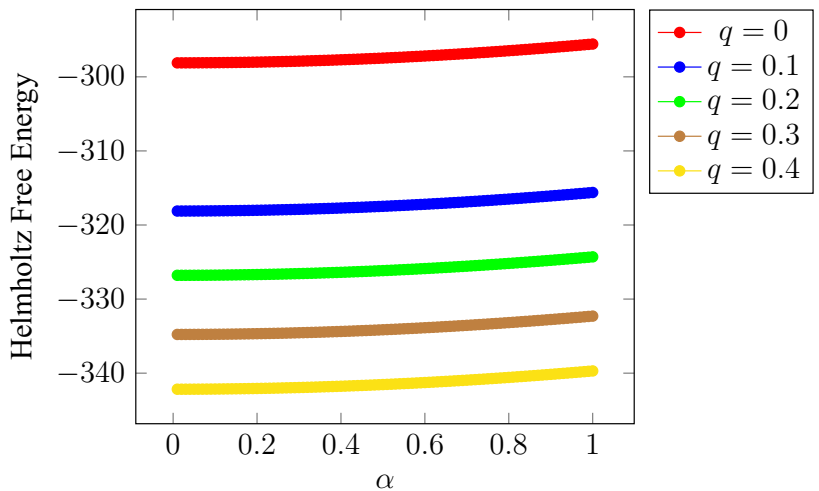

Fig. 9 Helmholtz free energy vs. $\alpha$ in the $q$-deformed superstatistics case considering $n=1, l=1, k=1, k_{B}=1, a=1$ and $T=100$ for different values of the deformation parameter

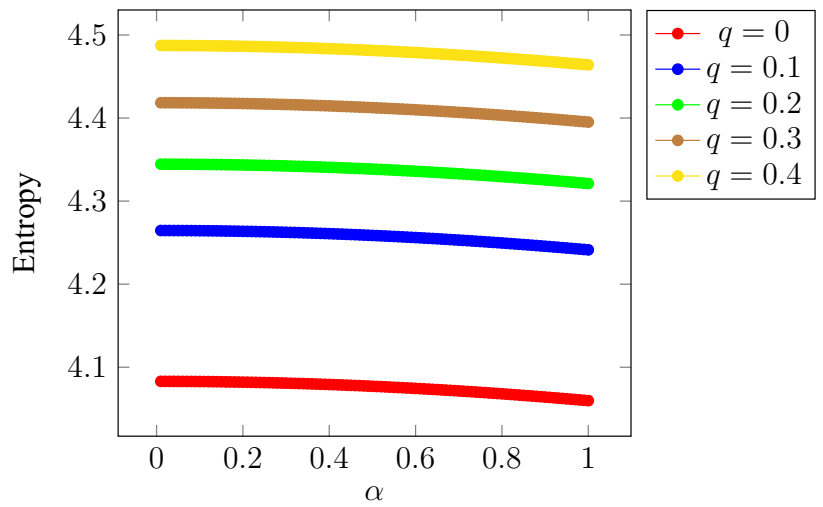

Fig. 10 Entropy free energy vs. $\alpha$ in the $q$-deformed superstatistics case considering $n=1, l=1, k=1, k_{B}=1, a=1$ and $T=100$ for different values of the deformation parameter

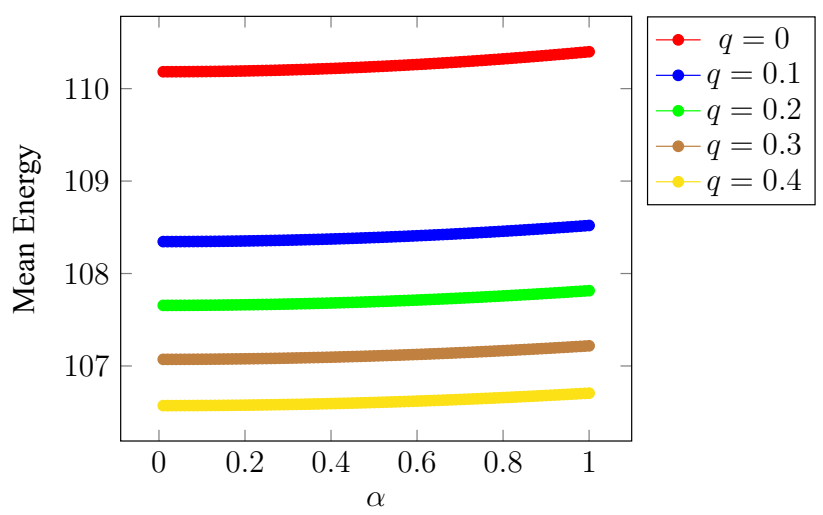

Fig. 11 Mean energy free energy vs. $\alpha$ in the $q$-deformed superstatistics case considering $n=1, l=1, k=1, k_{B}=1, a=1$ and $T=100$ for different values of the deformation parameter

deformation parameter, the specific heat at constant volume decreases for increasing $\alpha$ while in the presence of deformation is increasing. It is seen easily that, for vanishing of the modification, the treatments are repeated exactly as we faced in the previous subsection. 


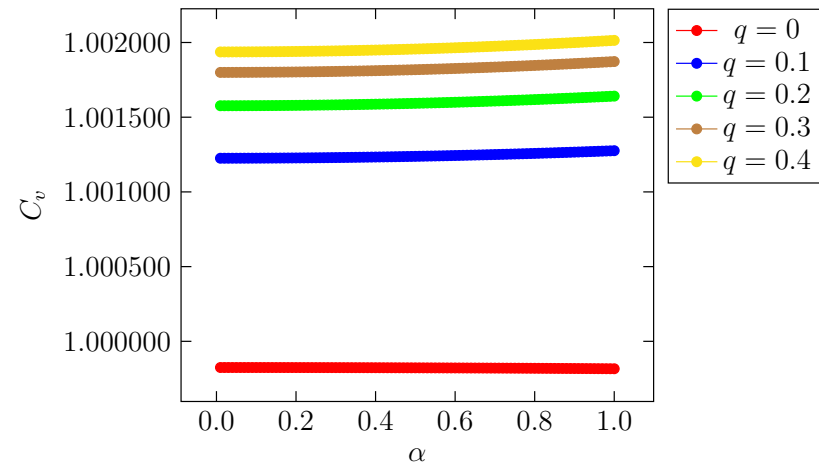

Fig. 12 Specific heat at constant volume vs. $\alpha$ in modified Dirac delta distribution case considering $n=3, l=1, k=1, k_{B}=1, a=1$ and $T=100$ for different values of the deformation parameter

\section{Conclusions}

In this article, we have studied the thermodynamical properties of the anharmonic oscillator within cosmic-string framework using ordinary and the $q$-deformed superstatistics. After an introduction of the superstatistics, we presented a new probability density using the Dirac delta function and its derivatives and then the effective Boltzmann factor was derived according to the new probability density. Next, we derived the Schrödinger equation within the cosmicstring framework. The wave function of the system considered was derived using the bi-confluent Heun function and the series form of the bi-confluent Heun functions. Having determined the spectrum, using the ordinary statistics approach, some of the thermodynamical properties for the ensemble of the considered system were derived. In the next step, we obtained once again these quantities according to the effective Boltzmann factor derived by the $q$-deformed superstatistics. Treatments of these quantities were illustrated graphically. It was shown that, for the $q$-deformed superstatistics case, by removing the modification parameter, the results of the ordinary statistics approach were derived.

Acknowledgements It is a great pleasure for the authors to thank the referee for the helpful comments.

Open Access This article is distributed under the terms of the Creative Commons Attribution 4.0 International License (http://creativecomm ons.org/licenses/by/4.0/), which permits unrestricted use, distribution, and reproduction in any medium, provided you give appropriate credit to the original author(s) and the source, provide a link to the Creative Commons license, and indicate if changes were made. Funded by SCOAP ${ }^{3}$.

\section{Appendix}

The differential equation of the bi-confluent Huen equation is [32]

$$
\begin{aligned}
& y^{\prime \prime}(x)+\left(\frac{1+\alpha^{\prime}}{x}+\beta^{\prime}-2 x\right) y^{\prime}(x) \\
& +\left(\gamma^{\prime}-\alpha^{\prime}-2-\frac{\delta^{\prime}+\left(1+\alpha^{\prime}\right) \beta^{\prime}}{2 x}\right) y(x)=0 .
\end{aligned}
$$

It should be noted that the notations used here are not related to the ones used within the text. Substituting Eq. (4.6) into Eq. (4.5), and after determination of the coefficients $A_{1}, B_{1}$ and $D_{1}$, we obtain

$$
\begin{gathered}
F^{\prime \prime}(y)+\left(\frac{2 A_{1}}{y}+2 B_{1}+4 D_{1}\right) F^{\prime}(y)+\left(\frac{2 A_{1} B_{1}+\frac{\varepsilon-k^{2}}{4}}{y}\right. \\
\left.+2 D_{1}\left(2 A_{1}+1\right)+B_{1}^{2}-\frac{a+l^{2} \alpha^{2}}{4}\right) F(y)=0 .
\end{gathered}
$$

Comparing Eqs. (A1) and (A2) shows that $D_{1}$ should be equal to $-1 / 2$ to produce the term $-2 x$ in the coefficient of the first derivative in Eq. (A1). Thus we have

$$
-\frac{1}{2}=-\frac{\sqrt{c_{v}}}{4} \rightarrow c_{\nu}=4
$$

\section{References}

1. T.W.B. Kibble, J. Phys. A 9, 1387 (1976)

2. A. Vilenkin, E.P.S. Shellard, Cosmic Strings and Other Topological Defects (Cambridge University Press, Cambridge, 1994)

3. S. Sarangi, S.-H.H. Tye, Phys. Lett. B 536, 185 (2002)

4. E.J. Copeland, R.C. Myers, J. Polchinski, JHEP 2004, 013 (2004)

5. R. Jeannerot, J. Rocher, M. Sakellariadou, Phys. Rev. D 68, 103514 (2003)

6. M. Landriau, E.P.S. Shellard, Phys. Rev. D 69, 023003 (2004)

7. P.A.R. Ade et al. (Planck), Astron. Astrophys. 571, A25 (2014)

8. A. Lazanu, P. Shellard, JCAP 1502, 024 (2015)

9. C. Ringeval, D. Yamauchi, J. Yokoyama, F.R. Bouchet, JCAP 1602, 033 (2016)

10. T. Charnock, A. Avgoustidis, E.J. Copeland, A. Moss, Phys. Rev. D 93, 123503 (2016)

11. T. Damour, A. Vilenkin, Phys. Rev. Lett. 85, 3761 (2000)

12. T. Damour, A. Vilenkin, Phys. Rev. D 71, 063510 (2005)

13. X. Siemens, V. Mandic, J. Creighton, Phys. Rev. Lett. 98, 111101 (2007)

14. S. Olmez, V. Mandic, X. Siemens, Phys. Rev. D 81, 104028 (2010)

15. P. Binetruy, A. Bohe, C. Caprini, J.-F. Dufaux, JCAP 1206, 027 (2012)

16. H. Vieira, V. Bezerra, G. Silva, Ann. Phys. 362, 576 (2015)

17. H. Cai, H. Yu, W. Zhou, Phys. Rev. D 92, 084062 (2015)

18. K. Jusufi, Gen. Relativ. Gravit. 47, 1 (2015)

19. A. de Pádua Santos, E.R.B. de Mello, Class. Quantum Gravity 32, $155001(2015)$

20. L.B. Castro, Eur. Phys. J. C 75, 287 (2015)

21. A. Beresnyak, Astrophys. J. 804, 121 (2015)

22. H. Hassanabadi, A. Afshardoost, S. Zarrinkamar, Ann. Phys. 356, $346(2015)$

23. F. Niedermann, R. Schneider, Phys. Rev. D 91, 064010 (2015)

24. M. Hosseinpour, H. Hassanabadi, Int. J. Mod. Phys. A 30, 1550124 (2015) 
25. M. de Montigny, M. Hosseinpour, H. Hassanabadi, Int. J. Mod. Phys. A 31, 1650191 (2016)

26. C. Becka, E.G.D. Cohen, Phys. A 322, 267 (2003)

27. S. Sargolzaeipor, H. Hassanabadi, W.S. Chung, Eur. Phys. J. Plus 133, 5 (2018)

28. C.R. Muniz, V.B. Bezerra, M.S. Cunha, Ann. Phys. 350, 105 (2014)
29. A.K. Dutta, R.S. Willey, J. Math. Phys. 29, 892 (1988)

30. N. Saad, R.L. Hall, H. Ciftci, J. Phys. A Math. Gen. 39, 8477 (2006)

31. H. Sobhani, A.N. Ikot, H. Hassanabadi, Eur. Phys. J. Plus 132, 240 (2017)

32. A. Ishkhanyan, K.A. Suominen, J. Phys. A Math. Gen. 34, 6301 (2001) 